CAHIERS DE

NARRATOLOGIE

\section{Cahiers de Narratologie}

Analyse et théorie narratives

25 | 2013

Humour et modernité dans les littératures de langues romanes du XIXe au XXIe siècle

\title{
Modernité et culture du rire
}

\section{Alain Vaillant}

\section{OpenEdition}

Journals

Édition électronique

URL : http://journals.openedition.org/narratologie/6774

DOI : 10.4000/narratologie.6774

ISSN : 1765-307X

Éditeur

LIRCES

Référence électronique

Alain Vaillant, « Modernité et culture du rire », Cahiers de Narratologie [En ligne], 25 | 2013, mis en ligne le 20 décembre 2013, consulté le 19 avril 2019. URL : http://journals.openedition.org/

narratologie/6774; DOI : 10.4000/narratologie.6774

Ce document a été généré automatiquement le 19 avril 2019

Article L.111-1 du Code de la propriété intellectuelle. 


\title{
Modernité et culture du rire
}

\author{
Alain Vaillant
}

\section{Préambules : questions de méthode et de vocabulaire}

1 Comme le titre l'indique, l'objectif est ici d'esquisser un essai de description et d'interprétation des formes et des modalités du rire, saisies dans leur relation avec l'époque moderne et, plus spécifiquement, avec ses caractéristiques politiques et sociales. En d'autres termes, il y sera question de poétique historique. La poétique historique, telle que je la définis et la pratique ${ }^{1}$, considère d'abord la littérature comme une pratique à la fois artisanale et artistique, dont l'histoire implique comme préalable celle de ses matériaux, de ses techniques et de ses traditions. Mais, outre la description précise des phénomènes textuels, elle exige de mobiliser un ensemble très divers de données historiques : apprentissages scolaires ou professionnels, poids des institutions littéraires, système des genres, état des techniques et des procédés, contraintes matérielles, etc. Elle suppose aussi, cela va sans dire mais c'est là sa principale difficulté, de se plonger dans de très vastes corpus textuels, sans souci de hiérarchie ou d'appréciation esthétique, mais pour mieux connaître, de l'intérieur et jusqu'aux processus cognitifs qui en découlent, l'horizon familier de tout écrivain et de tout lecteur. Enfin, parce que la littérature est une forme de communication parmi d'autres, la poétique historique doit s'inscrire dans une histoire générale des formes de communication qui, à chaque époque, sont amenées à interagir entre elles et à se modifier réciproquement : à cet égard, l'un des phénomènes les plus considérables propres au XIXe siècle européen est la constitution des industries de l'imprimé (presse et édition), qui bouleverse les conditions, économiques et sociales mais aussi formelles, de la production littéraire et qui, on le verra, a des conséquences immédiates sur la culture du rire.

2 Cependant, l'expression de "culture du rire " ne doit pas donner à penser que le rire serait un phénomène d'ordre culturel, ou historique. Il faut donc commencer en rappelant l'essentiel: le rire est une donnée anthropologique fondamentale, qui englobe et dépasse les apparences limitées dans le temps ou dans l'espace qu'il a pu adopter au 
cours de son histoire. Aussi socialisé et, pour ainsi dire, industrialisé qu'il semble parfois, le mécanisme comique plonge dans les zones les plus enfouies, les plus mystérieuses de l'homme. Dans l'inconscient que refoule le moi sérieux du sujet conscient, à coup sûr; toujours aussi, dans la mémoire des mondes merveilleux de l'enfance (le rire est nécessairement régressif, et c'est pourquoi il est profondément émouvant); plus généralement peut-être, dans un état archaïque et primitif de l'homme, enfoui quelque part dans les circonvolutions de l'esprit et l'épaisseur du corps, et auquel on n'a accès que dans les brefs instants de l'éclat de rire.

3 Mais cette universalité et cette permanence constituent aussi les principaux obstacles que rencontrent les analystes du rire. Spécialisation universitaire oblige, ceux-ci préfèrent donc s'attacher à des avatars du rire historiquement datés et déterminés. Qu'il s'agisse de la farce médiévale, de la satire classique, de l'« esprit » du XVIIIe siècle ou du grotesque des romantiques, l'objectif est alors non pas s'interroger sur le rire tel qu'en lui-même, mais de situer l'une de ses manifestations dans un contexte particulier, dont la compréhension est demandée à l'histoire des idées, de la culture ou de la littérature. On a pu ainsi travailler, de façon monographique et autonome, sur l'ironie, la blague ou la dérision en laissant de côté, comme de vagues évidences, la puissance et les motivations profondes du rire qu'elles déclenchent. C'est particulièrement vrai pour les recherches d'ordre linguistique ou stylistique qui, si elles échappent au cloisonnement séculaire, se gardent bien, du fait même de leur logique disciplinaire, de sortir de la stricte observation des faits discursifs et s'interdisent d'aborder le phénomène psychique du rire.

Enfin, le rire paraissant décidément insaisissable, on s'efforce d'en rendre compte en s'attachant à son usage et à sa signification morale ou idéologique plutôt qu'au matériau risible lui-même. Cette substitution ne souffre à peu près aucune exception : toute étude sur quelque production comique que ce soit (littéraire, théâtrale ou graphique) aboutit systématiquement à l'élucidation de ses enjeux sérieux (idéologiques, politiques, philosophiques), comme si le risible n'était qu'un ingrédient parmi d'autres et un simple instrument rhétorique, servant à amplifier l'efficacité argumentative du discours sousjacent à l'œuvre - à moins que, selon la version esthétisante de cette annexion du rire, la composante comique soit intégrée et subordonnée à un projet artistique supérieur. Qu'il s'agisse d'idéologie ou d'esthétique, on en arrive ainsi toujours, de façon plus ou moins directe et explicite, à la conclusion paradoxale que le rire vaut surtout par ce qui, en lui, resterait irréductiblement sérieux, donc non risible.

5 Prenant le contre-pied de ces orientations, mes travaux sur le rire ${ }^{2}$ partent d'un double postulat, dont ils s'efforcent de tirer toutes les conséquences formelles. D'une part, dans la mesure où le rire, selon toutes les théories admises et en empruntant le vocabulaire de la philosophie classique, est une "émotion » ou une " passion » sui generis, ils considèrent que, malgré la multiplicité de ses formes et la variabilité historique de ses manifestations culturelles, le rire est un, et c'est donc cette unité anthropologique essentielle qu'il s'agit de mieux comprendre. D'autre part, si le rire est bien une réalité spécifique, la culture du rire désigne tous les moyens élaborés pour faire rire, avec le plus de force et de plénitude possible, et non pas pour viser, au moyen du rire, un autre objectif - même si le rire, bien entendu, s'accompagne d'effets secondaires d'une efficacité souvent redoutable.

6 Mais il n'était évidemment pas possible d'aborder frontalement la question anthropologique du rire dans le cadre d'un article. Par « culture du rire », j'entends donc ici une culture structurée et reconnue comme telle, s'incarnant dans des formes et des pratiques fixes, reconnues, impliquant des métiers, des productions artistiques ou 
littéraires - en un mot, un marché du rire permanent, qui ne se constitue qu'à l'époque moderne.

7 Ce mot de «moderne ", qui fait couple avec celui de "modernité », a suscité tant de malentendus qu'il mérite lui aussi quelques explications liminaires. Les «temps modernes » désignent pour tous - pour les littéraires comme pour les historiens, quoi qu'on en dise parfois - la nouvelle période succédant à l'antiquité : elle est donc censée commencer soit à la Renaissance (le moyen âge apparaissant comme une période de transition) soit dès le moyen âge chrétien (c'est la périodisation adoptée par Hugo, dans la Préface de Cromwell). Quant au mot de "modernité », il apparaît dès le XVIIe siècle pour désigner, de façon neutre, le fait d'appartenir à l'époque moderne. En 1668, l'érudit Michel de Pure, évoquant certaines médailles d'apparence trop belle pour être antiques, parle à leur propos d'un « soupçon de modernité, s'il [lui] est permis de fabriquer ce mot $^{3}$ ». Il pense donc avoir forgé un néologisme, dont l'usage se répandra au XVIIIe siècle dans le vocabulaire archéologique - le plus souvent pour dénoncer d'éventuelles fausses antiquités.

8 En effet, les arts de l'Ancien Régime reposant en grande partie sur une imitation de l'antiquité, l'affirmation d'une spécificité de la culture moderne ne pouvait se faire qu'en termes mesurés et prudents, malgré la querelle des Anciens et des Modernes qui peut, à bon droit, apparaitre comme la première revendication d'une "modernité » avant la lettre. Le contexte change radicalement avec la Révolution française, qui tourne violemment (et à peu près définitivement, malgré la brève parenthèse de la Restauration), la page de la monarchie de droit divin, et, du même coup, celle du classicisme. La «modernité » ne signifie plus seulement le fait d'appartenir à l'époque moderne, mais qualifie la culture nouvelle, issue de l'après-Révolution, qui se pense désormais égale à l'Antiquité, voire supérieure à elle et, surtout, différente d'elle. Alors que la querelle des Anciens et des Modernes reposait sur une comparaison, donc une évaluation entre des cultures commensurables, la modernité postrévolutionnaire insinue l'idée d'une rupture radicale entre l'antique (ou ce faux antique qu'est le classique) et le moderne. Dès 1822, Balzac, dans un compte rendu anonyme et auto-promotionnel de son roman Le Centenaire, a joué un rôle décisif dans cette réinvention de la "modernité », qu'il écrit en italiques, comme pour signaler sa valeur de néologisme. Il emploie le mot à trois reprises. La première fois, pour expliciter le jeu de mots qu'implique le mot de modern/ité, construit sur le modèle vénérable de l'Antiqu/ité :

Après cette grande époque de l'espèce humaine, époque dont les bienfaits s'éteignirent avec les derniers feux de la bibliothèque d'Alexandrie, vint l'époque de la modernité.

9 La deuxième fois, pour condamner le classicisme, qui aurait sacrifié la modernité à sa volonté obstinée de singer l'antiquité :

Le résultat du siècle de Louis XIV fut cette doctrine vers laquelle l'esprit humain était invisiblement poussé ; et si la Grèce, en peignant des hommes, nous donna des héros, la première époque de la modernité, en voulant donner des héros, ne peignit point exactement l'homme.

La troisième fois, pour consacrer à la fois la modernité de «notre époque » (le XIXe siècle) et le genre du roman, «le seul qu'ait inventé la modernité ${ }^{4}$. Cette «modernité » renvoie donc au moment où, avec retard, les temps modernes prennent conscience d'eux-mêmes, et se revendiquent comme tels. Or, toute prise de conscience est progressive : il n'y a pas de sens à dater rigoureusement l'émergence de cette modernité, au terme d'un processus commencé, en toute hypothèse, dès le XVIIIe siècle. 
11 Une dernière étape est franchie après 1830, avec l'intronisation du Roi bourgeois (LouisPhilippe) et le triomphe du libéralisme économique, qui fait s'enfoncer l'Europe dans un mercantilisme industriel que la plupart des écrivains et des intellectuels condamnent. Désormais, l'époque parait si médiocre, si laide, si triviale et surtout si insignifiante qu'elle semble impropre à aucune sorte d'investissement esthétique. Alors que l'idéal de stabilité inhérent à l'âge antique ou classique donnait une consistance à son art, la culture née de la révolution industrielle est au contraire marquée par la folle succession des modes, l'absurde vacuité d'un temps vidé de sa substance historique. La modernité n'est plus la conscience lucide des temps modernes, mais la capacité culturelle de transmuer la laideur en beauté, selon une alchimie désormais réservée aux seuls vrais artistes. D’où la célèbre formule de Baudelaire : la "modernité ", c'est l'art "de dégager de la mode ce qu'elle peut contenir de poétique dans l'historique, de tirer l'éternel du transitoire ${ }^{5}$ ». La modernité baudelairienne radicalise celle de l'après-Révolution, mais elle n'en modifie pas la signification essentielle. La modernité baudelairienne n'est que le comble de l'utopie romantique, puisqu'elle part elle aussi en quête de sens et de beauté, comme le faisaient déjà Chateaubriand ou Lamartine, mais au royaume même de l'insignifiance et de la laideur, dès lors qu'il ne reste que lui : selon les termes d'une expression trouvée dès le Salon de 1846, elle permettra ainsi, malgré la médiocrité générale des existences ordinaires, d'accéder à "l'héroïsme de la vie moderne " et de comprendre sa beauté propre: car «la beauté absolue et éternelle n'existe pas, ou plutôt elle n'est qu'une abstraction écrémée à la surface des beautés diverses ${ }^{6}$ ». Toujours dans le Salon de 1846, Baudelaire écrivait dans les termes les plus explicites, en réponse à la question « Qu'est-ce que le romantisme? » : « Qui dit romantisme dit art moderne ${ }^{7} »$.

\section{Rire classique et rire moderne}

12 Cette mise en perspective de la modernité permet de mieux comprendre la nature et les évolutions du rire à l'époque moderne - c'est-à-dire, rappelons-le, depuis la Renaissance.

13 Commençons par un peu d'histoire sociale. La culture du rire telle que nous l'entendons, ayant ses modes d'expression et ses professionnels attitrés, implique l'existence d'un espace public autonome, au sens où l'entend Jürgen Habermas ${ }^{8}$. Pour Habermas, la notion d'espace public découle de deux distinctions fondamentales : d'une part entre le domaine public, qui correspond strictement à l'espace réservé à l'autorité publique de l'État, et le domaine privé ; d'autre part, à l'intérieur du domaine privé, entre la sphère privée, qui englobe à la fois les transactions économiques entre les personnes et le cercle familial, et ce qu'il appelle la sphère ou l'espace public, dévolu à l'échange intellectuel, culturel et politique.

14 Le domaine public, qui a en charge l'exercice de l'autorité et de la contrainte, ne peut absolument pas admettre le rire: par nécessité fonctionnelle, il est rigoureusement cantonné au sérieux. Le rire n'a donc le droit et la possibilité de se déployer que dans les limites du domaine privé. Bien sûr, il s'épanouit d'abord, à toute époque et quel que soit le mode d'organisation politique, dans la sphère privée (familiale et intime), satisfaisant le besoin anthropologique de rire. Néanmoins, l'épanouissement d'une culture du rire forte et permanente implique un espace public assez puissant et indépendant pour créer ses propres institutions culturelles et favoriser le déploiement de l'esprit de non-sérieux, sans pour autant mettre en danger le sérieux consubstantiel au domaine de l'autorité publique avec lequel il doit coexister. Or, l'épanouissement de cet espace public est 
directement lié, comme le note encore Habermas, au développement des échanges économiques, donc de la classe bourgeoise et des grandes cités marchandes. La naissance d'un capitalisme urbain (avec tous les phénomènes qui lui sont corrélés) a donc conditionné l'épanouissement du rire hors de l'espace privé : dans la Grèce antique, on riait davantage à Corinthe qu'à Thèbes, à Athènes qu'à Sparte. Pour l'Europe moderne, on sait que ce processus d'embourgeoisement est enclenché en Europe dès le XIIe siècle et accéléré à partir de la Renaissance : géographiquement, ce sont, parmi les principales puissances européennes, les États italiens puis, surtout, l'Angleterre et la France qui ont été les premiers à offrir les conditions socio-économiques de cette culture urbaine du rire - à la différence des pays encore trop exclusivement dominés par la féodalité rurale ou par l'autorité théocratique.

Cependant, au sein de ce concert européen du rire, la France occupe très tôt une position originale et privilégiée, si bien que la "gaieté " y apparaît comme le trait caractéristique du génie national, et reconnu comme tel par les observateurs étrangers : au XVIIIe siècle, l'esprit symbolise le haut degré de raffinement dont les élites s'enorgueillissent (en accablant au besoin de leur mépris le sérieux anglais ou la gravité germanique). Mais ce rire français n'est pas, comme on s'en doute, n'importe quel rire. Sous l'Ancien Régime, il a présenté trois traits caractéristiques remarquables, qui ont fini par faire souche dans la culture comique nationale et qui déborderont donc sur le XIXe siècle.

En premier lieu, alors que la France avait un tissu social assez comparable à l'Angleterre, elle n'a pas connu la même évolution vers le libéralisme et, au contraire, a vu l'instauration d'une monarchie absolue qui a renforcé considérablement le pouvoir de l'État. La contradiction était criante entre l'épanouissement social de la sphère publique et l'impuissance politique dans laquelle on la maintenait. Dans ces conditions, le rire a été doté d'une fonction à la fois de compensation et de contestation, qui explique la nature ouvertement idéologique de la tradition satirique française, très différente de l'humour d'autodérision qui, lui, s'est développé outre-Manche dès le XVIIIe siècle.

En outre, dans un pays où la religion était encore une religion d'État, la question du rire devait inévitablement croiser le fait religieux. Or, le catholicisme est à la différence du protestantisme une religion dogmatique s'appuyant sur son autorité spirituelle aussi bien temporelle et exigeant une soumission inconditionnelle à cette autorité. Elle n'offre pas d'autre alternative que l'acceptation ou le rejet: dans la monarchie française de droit divin, ce rejet ne pouvait se manifester que par l'effet indirect du rire - d'un rire qui était à la fois de provocation, de dérision et de profanation, porté par une violence anticléricale qui semble en effet propre au comique des pays de tradition catholique.

Enfin, l'aristocratie nobiliaire était la première lésée dans la mise sous tutelle de l'espace public par l'administration monarchique ; elle fut donc aussi la première à recourir aux ressources du rire, dont elle fit à la fois un instrument de résistance et un signe de distinction ; l'esprit est devenu l'une des composantes principales de l'ethos aristocratique. Davantage même : il s'est peu à peu imposé comme une seconde nature, au point que la simple disposition au rire spirituel a fini par se confondre totalement avec l'essence même de l'aristocratie. Indépendamment de son contenu, la capacité à rire, de tout et en toute circonstance, était censé démontrer la supériorité de la noblesse sur les autres forces sociales et, au-delà des frontières, celle de la nation française sur le reste de l'Europe.

Le rire français était donc à l'âge classique un rire contestataire et désacralisant, et il a gardé ces deux qualités, essentielles à l'aura libérale ou libertaire dont il jouit toujours 
dans l'opinion publique. Mais il était aussi un rire aristocratique : cet élitisme du bel esprit, en revanche, ne pouvait évidemment pas survivre à 1789. En fait, il lui est arrivé la même chose qu'à la grande cuisine ou aux autres privilèges de la haute aristocratie. On sait que les premiers restaurants gastronomiques ont ouvert, sous le Directoire, à l'initiative d'anciens maîtres cuisiniers naguère au service de grandes familles et mobilisant désormais leurs compétences pour leurs clients bourgeois. De même, tout se passe comme si le rire s'était déversé de la sphère aristocratique vers l'espace public révolutionné, évoluant d'un rire somme toute communautaire (même si cette communauté, la noblesse, était la deuxième caste du royaume) à un rire revendiquant cette fois son caractère proprement national.

20 Or, en se nationalisant, le rire français change radicalement d'allure. Le sourire ironique d'Ancien Régime, qui reposait sur l'étroite connivence, idéologique et culturelle, régnant au sein de la caste aristocratique, s'épanouissait au moyen de jeux d'esprit, d'allusions fines, de subtiles mystifications d'où étaient bannies, du moins en principe ${ }^{9}$, les formes réputées les plus basses du rire (les calembours et toutes les manipulations ludiques du langage). Au contraire, le rire public du XIXe siècle, qui s'adresse à un public beaucoup plus hétérogène, doit reposer sur des mécanismes rudimentaires, accessibles à des groupes sociaux qui ne maîtrisent pas forcément les mêmes savoirs. Il faut faire simple et efficace : triomphent donc le calembour, la charge parodique, la blague grivoise, voire obscène ou scatologique. Plus globalement, tous les jeux sur les signifiants et sur les codes eux-mêmes l'emportent sur les mécanismes liés aux seuls signifiés.

21 Cependant, n'exagérons pas l'hétérogénéité sociale des rieurs. Le public qui consomme le rire - qui a donc les moyens et le temps d'aller aux spectacles ou de lire les journaux - est majoritairement un public bourgeois, appartenant à cette nouvelle bourgeoisie issue de la Révolution et de la brusque redistribution des rangs et des fortunes qu'elle a permise. Rien n'est plus faux que l'image véhiculée par les écrivains eux-mêmes, de bourgeois qui seraient pénétrés de leur imperturbable gravité et en butte aux moqueries du milieu artiste. Balzac, Baudelaire, Gautier rient et persiflent non parce qu'ils détesteraient les bourgeois (pas seulement, du moins...), mais bien du fait même de leur appartenance à cette bourgeoisie si éloquemment méprisée. Le rieur au XIXe siècle est d'abord le Bourgeois et, s'il lui arrive de rire de lui-même ou de faire rire de lui, c'est qu'il le veut bien, précisément parce qu'il aime rire. Le succès de la presse pour rire, des caricatures, de la frivole littérature panoramique, prouve à lui seul l'importance de ce marché du rire. $\mathrm{Au}$ XIXe siècle, le public consomme massivement le rire, et ce rire reflète la doxa partagée par cette nouvelle bourgeoisie, à savoir à la fois un anticléricalisme moqueur à l'encontre d'une Église qui fait bloc derrière la droite légitimiste et un anti-idéalisme dont font les frais les romantiques et les utopistes de toute obédience.

Dans la France révolutionnée, c'est le sens de la blague, de la dérision gouailleuse dont le tout-Paris administre désormais les leçons au reste de l'Europe. Aujourd'hui encore, un parfum de légèreté, un second degré ironique toujours près d'affleurer singularisent une French touch qui irait de pair avec une forme inimitable d'élégante désinvolture. Déjà Stendhal, dans ses notes sur le rire, considérait comme une évidence que la France était "la patrie du rire» et que la "nation française", "vive, légère, souverainement vaniteuse [...] sembl[ait] faite exprès pour le rire ${ }^{10} »$.

23 Quant à l'écrivain, on mesure sans peine la difficulté à peu près insurmontable où l'entraîne sa haine du Bourgeois. Comment rire et faire rire d'un Bourgeois lui-même déjà en train de rire? Comment distinguer le rire bourgeois et la dérision artiste du rire 
bourgeois? Pire : dans la mesure où tout homme de plume (avec son éloquence et son lyrisme) est lui-même très souvent visé par le rire bourgeois, comment rire en retour d'un rire dont on est soi-même la victime ? À moins de croire à la distinction improbable d'un mauvais rire et d'un bon rire, pourquoi le rire serait-il louable ici, condamnable là ? Face au rire franc et massif du Bourgeois, la seule parade consiste en réalité à lui opposer ce rire au carré que représente le faux sérieux, cet art consommé de l'ambiguïté où le rieur se trouve pris à son propre piège, ne sachant plus ce dont il doit rire ou s'inquiéter, ou même s'il peut encore en rire. Le rire de l'écrivain moderne (celui d'un Flaubert ou d'un Baudelaire, par exemple) se signale donc, intrinsèquement, par sa nature originellement mystificatrice, par son pouvoir de constamment brouiller les cartes, de paraître sérieux au plus fort de la dérision ou, au contraire, de feindre la moquerie pour dire les choses les plus sincèrement graves.

\section{L'esthétique du rire moderne} romantisme ou à la modernité n'est pas séparable de cet envahissement de la culture du XIXe siècle par le comique sous toutes ses formes. À vrai dire, il n'est pas de grand écrivain, au moins à partir de 1830, c'est-à-dire avec l'abandon du premier romantisme, métaphysique et religieux, qui n'ait donné une place centrale au rire pour la conception et la réalisation de son œuvre. Je me contenterai ici d'évoquer les deux textes littéraires majeurs sur le rire, les seuls qui, en France, ont proposé une théorie explicite sur l'art du rire: la Préface de Cromwell de Hugo (1827) et l'essai De l'essence du rire de Baudelaire (1855).

Pour Hugo, qui résume ici la doctrine romantique héritée des Allemands, le rire moderne résulte de la conscience que l'homme chrétien a de sa dualité constitutive, de son être fait à la fois de matérialité et d'idéalité. De cette conscience découle une conception de la beauté qui repose, non plus sur la conformité à un modèle objectif, mais sur une nouvelle exigence de vérité, qui prenne le risque de l'hétérogénéité voire de le disharmonie et du contraste. De cette redéfinition du Beau découle une «forme » et un " type » nouveaux, respectivement la comédie et le grotesque. Le grotesque résulte donc de la complexité humaine ; plus précisément, il trahit et traduit la part charnelle de l'homme, sa nature matérielle. Pour la théologie chrétienne, c'est d'ailleurs parce que le rire vient de la chair, qui a failli depuis le péché originel, qu'il représente la part du diable, inquiétante et condamnable. Au contraire, pour Victor Hugo, la dualité, puisqu'elle a été voulue par Dieu et d'ailleurs incarnée en Jésus, est bonne et profondément chrétienne; elle révèle, non la faiblesse de l'homme, mais sa richesse ; et l'art (romantique) qui en découle est lui-même plus subtil, plus véritablement beau, plus profondément humain que l'art de la beauté simple. Plus encore: la chair elle-même, le monde corporel et physique est lui-même beau, puisqu'il a été voulu par Dieu. L'homme est chair et esprit, ombre et lumière, pesanteur et légèreté, toutes choses à égalité de valeur et de dignité, si bien que le grotesque n'est rien d'autre que la conséquence esthétique de ce principe d'égalité.

Cette dignité égale du matériel et du spirituel se traduit artistiquement chez Hugo par son goût immodéré pour tout ce qui a une forte présence sensorielle; pour les couleurs vives, les matières épaisses, les bruits (aussi discordants que possible) ; pour tout ce qui signale que la matière existe, heureuse, insolente, épanouie, mais émouvante aussi. Les trivialités, les incongruités prétendument choquantes, les énumérations (à la fois si flamboyantes et 
si pesantes), les effets insistants d'allitérations qui remplissent la bouche du diseur de vers, ne sont pas que des provocations, des défis au bon goût, mais reflètent un bonheur charnel qui a gardé l'essentiel de la leçon épicurienne et qui se souvient de l'héritage rabelaisien. La référence à Rabelais s'impose en effet lorsqu'il est question de romantisme. Car le moyen âge a révélé que le comique du corps grotesque n'était pas un comique purement abstrait, une simple fantaisie. Dans la tourmente historique qui ébranle l'Europe médiévale, il devient le comique des peuples souffrants, malmenés, sacrifiés à la violence des puissants.

Aussi le rire romantique est-il par essence politique et démocratique, même s'il l'ignore lui-même parfois et qu'il tourne son esprit de contestation en morale du mépris. En effet, puisque le corporel grotesque est assimilé aux faibles et aux malheureux, l'opposition axiologique du sérieux et du risible, du spirituel et du matériel, du haut et du bas est non seulement absurde, mais devient moralement insupportable. Au contraire, le rire vise à rapprocher fraternellement le haut et le bas - donc, par une incessante dialectique, à rabaisser le haut et à élever le bas. Cependant, si cette esthétique consiste en une perpétuelle inversion du haut et du bas, la visée éthique de cette esthétique est, bien entendu, que, pour tous, le haut finisse par l'emporter sur le bas, que tous les corps (et d'abord ce bas politique que représente le peuple) se spiritualisent, une fois que les esprits auront appris à faire bon accueil aux corps et à les aimer.

Baudelaire, dans son essai De l'essence du rire (1855), parachève la doctrine romantique du rire, dont Victor Hugo avait jeté les bases en 1827, mais de surcroît, en privant le rire de l'optimisme politique auquel Hugo ne renoncera jamais, lui assigne par compensation la mission artistique la plus haute qu'on ait jamais songé lui confier. Le point de départ est, chez lui aussi, métaphysique. Le rire procède, là encore, de la nature composite de l'homme. L'homme est double, à la fois esprit et corps, et la conscience de cette dualité le met à l'écart de la nature, l'empêche de communier pleinement avec l'harmonie universelle. L'homme rit de se savoir supérieur à la nature, qui est simple. Ou plutôt, l'homme ne rit pas de se savoir, mais de se croire supérieur. Comme il est duel, il est conscient que, si une partie de lui (la corporelle) est faible et misérable, l'autre (la spirituelle) lui confère cependant une force qui lui est propre. Le processus psychologique $\mathrm{du}$ rire vient de la conflagration entre ces deux intimes convictions. Au moment où il se désole de sa faiblesse, il éprouve un brusque soulagement à se sentir, malgré tout, doué d'une force singulière, et il éclate alors de rire. Ce rire de supériorité, malgré des similitudes apparentes, doit donc être absolument distingué du rire de la joie, par lequel l'être pur (l'enfant, par exemple) manifeste la conscience heureuse de son unité et sa participation non problématique, pour ainsi dire végétale, à la simplicité de nature et aux forces vives de l'univers.

Ainsi, l'homme qui rit se sent supérieur. Encore faut-il s'entendre sur l'objet de ce sentiment de supériorité : c'est ici que Baudelaire introduit une alternative capitale. De deux choses l'une, donc.

L'homme peut s'estimer supérieur aux autres hommes : il rit alors d'imaginer (ou de voir représenter) ses semblables plus bêtes, plus vicieux qu'il ne se conçoit lui-même. Ce rire est le comique selon Aristote, le comique d'avant le romantisme, que Baudelaire nomme " comique ordinaire ». C'est un comique de nature inférieure, pour trois raisons. Tout d'abord, «le comique est, au point de vue artistique, une imitation ${ }^{11}$ » et n'exige aucune faculté créatrice : il est à ce titre aussi méprisable que le mauvais réalisme qui se contente de copier le réel. Ensuite, il est encore plus méprisable que ce réalisme dévoyé parce qu'il 
imite en enlaidissant, afin de provoquer le rire: non seulement il ne produit pas de beauté, mais il surenchérit sur la laideur du monde. Enfin, ce comique, toujours utilisé dans une logique satirique, sert indirectement - par les moyens du rire - à montrer le chemin de la morale : coupable de vouloir enseigner et rectifier, il instrumentalise le rire pour le mettre au service du sérieux et pour le dévoyer. Baudelaire appelle donc indifféremment le comique ordinaire " comique significatif », dont la simplicité explique son succès de mauvais aloi. Les deux représentants les plus illustres du "comique ordinaire » sont Molière et Voltaire : autant dire de tout l'héritage classique du comique. Mais l'homme peut aussi se penser supérieur, non aux autres hommes, mais à la nature elle-même. Il prouve alors cette supériorité en refusant d'imiter la nature, en partant d'éléments naturels pour aboutir, grâce à sa puissance imaginative, à de vraies créations. Ce comique-là - qu'il appelle d'abord "grotesque » comme Hugo puis, pour mieux dire, « comique absolu » - combine le fantastique et la fantaisie, la puissance du rêve et la force du rire.

Le processus du comique absolu est double : d'abord partir « d'éléments préexistants de la nature ${ }^{12} »-$ ce qui suppose une intime compréhension de cette nature et de ses mécanismes profonds -, puis une composition imaginaire, qui met en branle l'instinct de supériorité, mais à des fins créatrices et pour un résultat jubilatoire : cette dialectique de l'imagination et du rire recouvre exactement le jeu du "surnaturalisme» et de l'« ironie », que Baudelaire désigne comme les "deux qualités littéraires fondamentales ${ }^{13}$ ». Le comique absolu, parce qu'il manifeste le désir de l'homme de s'extraire des contraintes naturelles et sa volonté de libre création, s'identifie totalement au romantisme, tel que le conçoit Baudelaire. Le rire n'est plus une simple composante de l'esthétique, auquel cas il serait loisible à des critiques ayant fait le choix du sérieux de l'ignorer; il constitue la substance même de l'art, du moins lorsque celui-ci atteint la région du génie et de l'absolu :

L'essence très relevée du comique absolu en fait l'apanage des artistes supérieurs qui ont en eux la réceptibilité suffisante de toute idée absolue ${ }^{14}$.

Le « comique absolu » permet d'accéder à l'art supérieur parce qu'il libère l'imagination, rend l'esprit accessible, avec une intensité extraordinaire, à toutes les émotions sensibles, crée les conditions psychiques de l'invention artistique. Baudelaire se prend même à rêver. Il est vrai que le rire, marque de supériorité, reste toujours entaché par le dualisme de l'homme :

J'ai dit : comique absolu ; il faut toutefois prendre garde. Au point de vue de l'absolu définitif, il n'y a plus que la joie. Le comique ne peut être absolu que relativement à l'humanité déchue, et c'est ainsi que je l'entends ${ }^{15}$.

Pourtant, si l'homme pouvait, asymptotiquement, se rapprocher de la joie heureuse de l'enfant, de la pure extase de l'unité restaurée, ce ne pourrait être que l'artiste doué du génie du vrai rire :

[...] le rire causé par le grotesque a en soi quelque chose de profond, d'axiomatique et de primitif qui se rapproche beaucoup plus de la vie innocente et de la joie absolue que le rire causé par le comique de mœurs ${ }^{16}$.

Alors, par la grâce du rire, l'artiste pourra accéder au génie, c'est-à-dire à la joie de l'enfant, à l'ivresse surnaturaliste de l' " enfance retrouvée à volonté ${ }^{17}$ ", que Baudelaire évoque dans Le Peintre de la vie moderne. 


\section{Le lyrisme de l'ironie moderne}

35 ironique et mystificateur que pratiquait avec délices Baudelaire. Pourtant, à mesure que le siècle avance et que l'idéalisme se brise sur le sol rude des réalités, tous les écrivains découvrent progressivement que le lyrisme, s'il veut durer et rester conséquent avec luimême, a le besoin vital de l'ironie et que, réciproquement, l'ironie doit se muer en lyrisme, pour ne pas se réduire à une simple pose mystificatrice. Sans ironie, le lyrisme, s'il reste porté par le rêve illusoire d'une harmonie universelle, est constamment menacé par le reflux de la désillusion, par la révélation de sa propre imposture, par le renoncement définitif. Et l'on risque alors de tomber de très haut. En 1848, le poète Lamartine, chef du gouvernement provisoire de la France en révolution, fait partager à la nation entière - qu'il refonde alors en république - l'illusion lyrique d'un bonheur à portée de main. Le 10 décembre 1848, il se présente aux premières élections présidentielles, au suffrage universel, et rassemble sur son nom le nombre ridicule de 21032 voix ( $0,28 \%$ des votes) : au même moment, le lyrisme lamartinien est foudroyé et disparaît de la scène politique et poétique. Au contraire, le lyrisme artistique des ironistes de la modernité prend corps après leur désillusion face aux défaillances de l'Histoire, et du fait de cette désillusion : il sera donc indestructible.

C'est Flaubert qui fournit les analyses les plus claires de ce lyrisme étrangement ironique, où le burlesque doit finir par faire pleurer d'émotion - comme dans Madame Bovary dont il commente ainsi la gestation à Louise Colet, le 9 octobre 1852 :

Voilà deux ou trois jours que ça va bien. Je suis à faire une conversation d'un jeune homme et d'une jeune dame sur la littérature, la mer, les montagnes, la musique, tous les sujets poétiques enfin. - On pourrait la prendre au sérieux, et elle est d'une intention de grotesque. Ce sera, je crois, la première fois qu'on verra un livre qui se moque de sa jeune première et de son jeune premier. L'ironie n'enlève rien au pathétique. Elle l'outre, au contraire. - Dans ma troisième partie, qui sera pleine de choses farces, je veux qu'on pleure ${ }^{18}$.

Pleurer des " choses farces ", c'est aussi ce qu'il appelle, dans une lettre écrite deux jours avant, adopter le " point de vue d'une blague supérieure, c'est-à-dire comme le bon Dieu les voit $^{19}{ }^{\prime}$. Ou c'est encore, comme il se caricature cette fois le 8 mai 1852, pleurer d'émotion face aux autres et à leurs souffrances mais, dans le même temps, rire de soi-même en se regardant pleurer dans une glace :

Personne plus que moi n'a au contraire aspiré les autres. J'ai été humer des fumiers inconnus, j'ai eu compassion de bien des choses où ne s'attendrissaient pas les gens sensibles. - Si la Bovary vaut quelque chose, ce livre ne manquera pas de cœur. L'ironie pourtant me semble dominer la vie. - D'où vient que, quand je pleurais, j'ai été souvent me regarder dans la glace pour me voir? - Cette disposition à planer sur soi-même est peut-être la source de toute vertu ${ }^{20}$.

On peut douter des vertus réellement morales de cette force d'ironisation. Mais elle fournit la clé de ce sentimentalisme paradoxal, qu'une dernière expression, dans la même lettre, condense parfaitement, le « lyrisme dans la blague » :

Le comique arrivé à l'extrême, le comique qui ne fait pas rire, le lyrisme dans la blague, est pour moi tout ce qui me fait le plus envie comme écrivain [...].

Car seul ce sens de la blague permet à l'artiste d'allier indissolublement les deux tendances qu'il se doit de concilier, sous peine de se disqualifier, l'exigence de lucidité 
critique à l'égard d'un monde où il ne reconnaît plus le reflet de ce qu'il aime et, malgré tout, une capacité émotionnelle intacte (voire amplifiée par sa désillusion) qui le porte à embrasser cette réalité toujours désirable et à se l'absorber. Les écrivains «modernes " du XIXe siècle sont ainsi les premiers à avoir fait du rire le médium privilégié de la communication lyrique. Le rire est en effet un instrument d'une puissance inouïe, puisqu'il fait immédiatement comprendre et partager, avec le minimum de mots et par la seule force des images incongrues qui le suscitent, une vision du monde personnelle et complexe : ce qui nous ramène, in fine, à la figure du sujet.

Comme le note Baudelaire le rire est toujours dans le rieur, non dans la chose risible. Si le rieur rit de quelque chose, c'est que, derrière cette chose, il sait ou soupçonne la présence d'un autre rieur pour lequel il éprouve un sentiment diffus de complicité. Le rire impose le principe d'une relation interpersonnelle et la reconnaissance de l'autre : on rit toujours avec quelqu'un. Tout rire est rire de connivence : même lorsqu'on rit seul, on rit de se savoir rire, une part de soi se réjouissant du rire de l'autre part, et le rire se nourrit de la jouissance ressentie à ce dédoublement solitaire. Dans le cas d'un rire ironique, l'œuvre comique enclenche un processus qui conduit nécessairement à s'imaginer le rieur derrière le trait ironique, le sujet écrivant derrière l'allusion intertextuelle, le procédé linguistique ou la figure rhétorique.

41 Ce lyrisme du rire installe l'auteur sur une ligne de crête où il est extraordinairement difficile de se tenir. L'authentique émotion lyrique reste son horizon, toujours désirable et jamais atteignable, mais le franc abandon à l'illusion lyrique en serait la négation même. Néanmoins, l'ironie ne doit pas s'y manifester par une franche dérision ni par une drôlerie explicite, mais par le sentiment distillé au fil des pages que rien n'est si clair ni si simple qu'il n'y paraît. Au-delà des émotions exprimées noir sur blanc, l'œuvre diffuse une vague impression de facticité, un ordre curieusement concerté dont les incongruités calculées ne suffisent pas à déclencher le rire et à rompre le charme, mais entretiennent le soupçon qu'une vague inconvenance se trame en coulisses - coulisses où l'auteur ne cesse de feindre d'inviter le lecteur, tout en multipliant les chausses trappes pour lui en interdire l'accès. Si bien que la sourde présence de cette ironie auctoriale finit par accroître l'émotion lyrique, en lui prêtant une profondeur à tout jamais insondable. On conçoit que, d'un tel idéal esthétique, qui est peut-être la plus belle réussite de la modernité, on n'a pu par instants s'en approcher qu'aux périodes où l'Histoire elle-même bascule de l'enthousiasme à la mélancolie et où l'écrivain est contraint de s'abandonner totalement à son hyperesthésie artistique - par exemple, pour la France du XIXe siècle, vers 1857, au plus fort du Second Empire, alors que l'espérance démocratique a provisoirement déserté la scène de la politique et que la société est emportée, définitivement cette fois, dans l'emballement industriel et consumériste des économies occidentales. 


\section{NOTES}

1. Pour une présentation théorique générale de la poétique historique, voir Alain Vaillant, L'Histoire littéraire, Paris, Colin, « U », 2009.

2. Le présent article synthétise en effet plusieurs de mes travaux sur le rire, dont il reprend parfois les analyses de façon partielle ou adaptée. Voir, en particulier, Baudelaire poète comique, Rennes, PUR, 2006 et Le Veau de Flaubert, Paris, Hermann, 2013, ainsi que deux ouvrages collectifs que j'ai récemment dirigés: L'Esthétique du rire, Nanterre, Presses de Paris Ouest, "Orbis litterarum ", 2012 ; Le Rire moderne, Nanterre, Presses de Paris Ouest, 2013.

3. Michel de Pure, Idée des spectacles anciens et nouveaux, Paris, Brunet, 1668, p. 5. Cette citation ainsi que les analyses lexicales qui l'accompagne sont empruntées à: René Fest, Petite Étude lexicologique du mot "modernité», mémoire de master (dir. Alain Vaillant), Nanterre, université Paris Ouest, 2013.

4. La redécouverte de cet article de 1822 est due à Stéphane Vachon, à qui nous empruntons également les passages cités: voir Stéphane Vachon, "Honoré de Balzac a inventé la modernité », dans Paratextes balzaciens. "La Comédie humaine" en ses marges, R. Le Huenen et A. Oliver éd., Toronto, Centre d'études du XIXe siècle Joseph Sablé, 2007, p. 205-220.

5. Charles Baudelaire, Le Peintre de la vie moderne, dans Euvres complètes, C. Pichois éd., t. 2, Paris, Gallimard, «Bibliothèque de la Pléiade », 1976, p. 694.

6. Charles Baudelaire, Salon de 1846, dans Euvres complètes, t. 2, ouvr. cit., p. 493.

7. Ibid., p. 421.

8. Voir Jürgen Habermas, L'Espace public. Archéologie de la publicité comme dimension constitutive de la société bourgeoise, Paris, Payot, 1992 (éd. or. 1962).

9. Sur les formes plus franches et moins policées du rire aristocratique, voir Antoine de Baecque, Les Éclats du rire : la culture des rieurs au XVIIIe siècle, Paris, Calmann-Lévy, 2000.

10. Stendhal, Du rire, Antoine de Baecque éd., Paris, Rivages, 2005, p. 90-91.

11. Charles Baudelaire, «De l'essence du rire " (1855), dans Euvres complètes, Claude Pichois éd., Paris, Pléiade, tome 2, 1976, p. 535.

12. Charles Baudelaire, «De l'essence du rire » (1855), dans Euvres complètes, ouvr. cité, p. 535.

13. Charles Baudelaire, Fusées, dans Euvres complètes, Claude Pichois éd., t. 1, Paris, Galiimard, «Bibliothèque de la Pléiade », 1975.

14. Charles Baudelaire, «De l'essence du rire» (1855), dans CEuvres complètes, t. 1, ouvr. cité, p. 536.

15. Ibid.

16. Ibid., p. 535.

17. Charles Baudelaire, Le Peintre de la vie moderne, dans Euvres complètes, t. 2, ouvr. cité, p. 690.

18. Gustave Flaubert, Correspondance, t. 2, Jean Bruneau éd., Paris, Gallimard, « Bibliothèque de la Pléiade ", 1980, p. 172.

19. Ibid., p. 168.

20. Ibid., p. 84-85. 


\section{RÉSUMÉS}

Dans le cadre d'une poétique historique des formes littéraires, l'article se propose d'analyser la singularité du rire dans la modernité du XIXe siècle, d'en expliquer l'émergence dans le contexte de l'après-Révolution et d'en préciser les liens avec la culture médiatique dont Paris devient alors le centre. Un bref commentaire des deux grands textes majeurs sur le rire de l'époque (la Préface de Cromwell de Hugo et De l'essence du rire de Baudelaire) permettra aussi de mesurer l'invention proprement littéraire dont le rire est alors à la fois le moyen et la fin. Grâce à cette redéfinition esthétique du rire, le comique se confond avec le lyrisme - avec un lyrisme ironisé qui est la principale contribution de la modernité à l'art du rire.

As part of the historical poetics of literary forms, this article intends to analyse the singularity of laughter in nineteenth-century French "modernité " (modernity), to explain how it appeared after the French revolution and to precise its links with the media-centered culture whose center becomes at this time Paris. A short commentary of the two main French literary texts about laughter (the Preface de "Cromwell" by Victor Hugo and the essay De l'essence du rire by Charles Baudelaire) will also enable to estimate the truly literary invention in which laughter is at the same time the means and the aim. Thanks to this esthetical redefinition of laughter, the comical merges with lyricism - with an ironical lyricism which is the main contribution of the modernity to the art of laughter.

\section{INDEX}

Index géographique : France

Index chronologique : XIXe siècle

Mots-clés : rire, ironie, romantisme, modernité, grotesque, comique, histoire littéraire

\section{AUTEUR}

\section{ALAIN VAILLANT}

Alain Vaillant est professeur de littérature française à l'université Paris Ouest et directeur du Centre des sciences de la littérature française (CSLF). Il est en outre directeur de la revue Romantisme. Il est spécialiste du romantisme, de poétique historique, d'histoire de la poésie et d'histoire des institutions littéraires au XIXe siècle (en particulier, de la presse et de l'édition) plus généralement, il est un théoricien de l'histoire littéraire. Ses principaux ouvrages sont : La Poésie, Paris, Colin, 1992 (2 ${ }^{\mathrm{ème}}$ éd. 2008) ; Histoire de la littérature française du XIXe siècle (en collaboration avec Jean-Pierre Bertrand et Philippe Régnier), Rennes, PUR, 1998 ( 2 ème éd. 2007) ; 1836. L'an 1 de l'ère médiatique (en collaboration avec Marie-Ève Thérenty), Paris, Nouveau Monde éditions, 2001 ; L'Amour-fiction. Discours amoureux et poétique du roman moderne, Saint-Denis, Presses universitaires de Vincennes, 2002 ; La Crise de la littérature. Romantisme et modernité, Grenoble, ELLUG, 2005 ; Baudelaire poète comique, Rennes, PUR, 2007 ; L'Histoire littéraire, Paris, 
Colin, coll. «U », 2010 ; Baudelaire journaliste (anthologie), Paris, GF-Flammarion, 2011 ; Le Veau de Flaubert (Paris, Hermann, automne 2013). Il a dirigé une douzaine d'ouvrages collectifs : tout dernièrement, La Civilisation du Journal, histoire culturelle et littéraire de la presse au XIXe siècle (avec Dominique Kalifa, Philippe Régnier et Marie-Ève Thérenty, chez Nouveau Monde éditions, 2011), le Dictionnaire du romantisme (CNRS éditions, 2012), L'Esthétique du rire (Presses universitaires de Paris Ouest, 2012), Le Rire moderne (Presses universitaires de Paris Ouest, 2013). 